\title{
The Sampling Theorem based Methodology for Harmonics Analysis
}

\author{
P. Bokoro ${ }^{1}$, J. Pretorius $^{2}$ and M. Case ${ }^{1}$ \\ ${ }^{1}$ Department of Electrical \& Electronic Engineering Technology \\ University of Johannesburg \\ Doornfontein Campus- Johannesburg, 2028 (South Africa) \\ Phone/Fax number: +0027 559 6114/6119, e-mail: pitshoub@uj.ac.za,mcase@uj.ac.za \\ ${ }^{2}$ Department of Electrical \& Electronic Engineering Science \\ University of Johannesburg \\ Auckland Park Campus- Johannesburg 2006, (South Africa) \\ Phone/Fax number: +0027 559 3377/4947, e-mail: jhcpretorius@uj.ac.za
}

\begin{abstract}
This paper investigates the validity of the sampling theorem in the analysis of supply side line current harmonics as applied to 6-pulse AC-DC thyristor converters. The common pattern of the percentage harmonic factor, for the converter characteristic harmonic current, per incremental change of the thyristor firing angle is used as the basis of the study. The RMS values of the peak amplitudes of the characteristic harmonic currents induced in the input-phase of the converter supplying a DC motor are described in terms of the sampling theorem equations and further evaluated in terms of their respective individual harmonic contributions as the thyristor firing angle is changed. The MatLab/Simulink package is also employed to simulate the forward drive speed control operation of a DC motor using the 6-pulse AC-DC thyristor converter. However the percentage harmonic factor trends observed in the sampling theorem method appear to be generally increasing which is in contradiction to the MatLab/Simulink produced trends.
\end{abstract}

\section{Key words}

Sampling theorem, line current harmonics, thyristor converter, firing angle, harmonic factor.

\section{Introduction}

One of the most efficient and modern ways of statically achieving bidirectional power flow is the use of thyristor converters [1]. This however comes at the cost of current harmonics being generated at the input lines of the static converter. The control technique of these converters is essentially based on incremental or decremental adjustments of the thyristor firing angle [2]. This inevitably makes the firing angle such a considerable factor in the analytical description of line current harmonics.

Most of harmonic analysis techniques suggested in the literature generally consist of algorithm implementation or iterative procedures requiring harmonic sources to be modelled either in time-domain, in frequency-domain or in harmonic-domain, and seem to come short of sufficient description of converter control parameters such as the firing, commutation or overlap angles [3]. However the formulation of the sampling theorem method establishes the link between the converter control parameters and the magnitude of current harmonics induced back to the input phases. It is known that the magnitude of the lower order harmonics of current generated in the input lines of controlled converters increases as the firing angle is increased [4]. This should mean a decrease in terms of the percentage harmonic factor (HF) as the firing angle increases. This knowledge is exploited to test the adequacy of the sampling theorem method in the study of harmonic current components generated in the supply side lines of the converter.

This work therefore endeavours to use the sampling theorem method for describing the 6-pulse AC-DC thyristor converter input phase current in terms of the fundamental and the respective characteristic harmonic components, to produce the trends obtained by observing the percentage harmonic factor (HF) per incremental change of the firing angle, and to simulate the forward drive speed control of a DC motor using a 6-pulse ACDC thyristor converter with the MatLab/Simulink package.

However the percentage HF obtained generally appears to be increasing as the firing angle is increased which seems to be contradictory to the trends obtained by simulation where linear decay in the percentage HF is noted as the firing angle is increasingly adjusted.

\section{Sampling Theorem}

The sampling theorem is sometimes referred to as the Nyquist- Shannon theorem. It suggests that a continuoustime band limited signal that has been sampled can be accurately reconstructed from an infinite sequence of samples if the sampling rate exceeds $2 f_{h}$ samples per second, where $f_{h}$ is the highest frequency in the 
continuous-time signal [6]. The sampling theorem essentially consists of two fundamental steps: the sampling and the reconstruction processes.

\section{A. Sampling Process}

A continuous-time band limited signal or function $x(t)$ is uniformly sampled at the sampling rate $f_{s}>$ $2 f_{h}$ to form a discrete-time signal or function $x_{T}(n)$, whose sequence values $x(n)$ are the samples of the continuous-time function $x(t)$. This process can be mathematically described in terms of the product of the infinite series of samples of the continuous-time function to the impulse function $\delta(t-n T)$. This therefore yields equation (1) given below.

$$
x_{T}(n)=\sum_{n=-\infty}^{\infty} x(n) \cdot \delta(t-n T)
$$

Where $\left(T=\frac{1}{f_{s}}\right)$ is the sample spacing.

The relationship obtained in (1) could further be rewritten in terms of the frequency spectrum equation given in (2).

$$
X_{d}(f)=\sum_{n=-\infty}^{\infty} f_{s} \cdot X\left(f-n f_{s}\right)
$$

Where $X_{d}(f)$ is the frequency spectrum of the discretetime function $x_{T}(n)$, which is obtained by using the discrete-time Fourier transform (DTFT).

\section{B. Reconstruction or Interpolation Process}

The continuous-time signal $x(t)$ can be reconstructed from the discrete samples of the discrete-time signal $x_{T}(n)$ provided that the sampling rate exceeds twice the highest frequency of the continuous-time signal or function. Mathematically, this process can be expressed as the product of the discrete-time signal or function $x_{T}(n)$ to the sine cardinal (sinc) function. This is given in equation (3).

$$
x(t)=x_{T}(n) \cdot \frac{\sin \pi\left(f_{s} t-n\right)}{\pi\left(f_{s} t-n\right)}=\sum_{n=-\infty}^{\infty} x(n) \cdot \frac{\sin \pi\left(f_{s} t-n\right)}{\pi\left(f_{s} t-n\right)}
$$

Equation (3) holds true provided that the NyquistShannon condition is met:

$$
f_{s}>2 f_{h}
$$

This enables each term of equation (3) to be rewritten in terms of its frequency spectrum, which is obtained by applying Fourier transform (FT). Equation (3) would therefore become:

$$
X(f)=X_{d}(f) \cdot S_{i}
$$

Where $X_{d}(f)$ and $S_{i}$ are the frequency spectrums of the discrete-time domain signal and the sine cardinal function respectively. Equation (4) is commonly employed in digital signal processing (DSP) applications where analogue signals are transmitted using digital techniques for the purpose of filtering the noise level associated to the transmitted analogue signal [7]. In this context, $X_{d}(f)$ is the modulating signal, $S_{i}$ is the carrier signal and $X(f)$ is the spectrum of the original signal to be reconstructed.

\section{Line Current Harmonic Analysis}

Thyristor converters predominantly generate $(m p \pm 1)$ harmonic current components back to the input lines [8]. The factor $m$ is any multiple integers and $p$ is the converter pulse number. Therefore for a 6-pulse converter the following characteristic harmonics are expected: $5^{\text {th }}, 7^{\text {th }}, 11^{\text {th }}, 13^{\text {th }}, 17^{\text {th }} \ldots$ In converter applications such as the DC variable speed drive (VSD) the firing angle is relied upon to provide the control means of the motor output voltage and subsequently that of the speed as well. To apply the sampling theorem technique to the 6-pulse AC-DC thyristor converter the DC output current, which could be expressed as frequency spectrum equation, as well as the thyristor control signal (the train pulse), whose equation is analogous to the FT equation of the sinc function, are regarded as the modulating and the carrier signals respectively. The distorted input current, whose frequency spectrum equation can be reconstructed, is perceived as the signal to be transmitted.

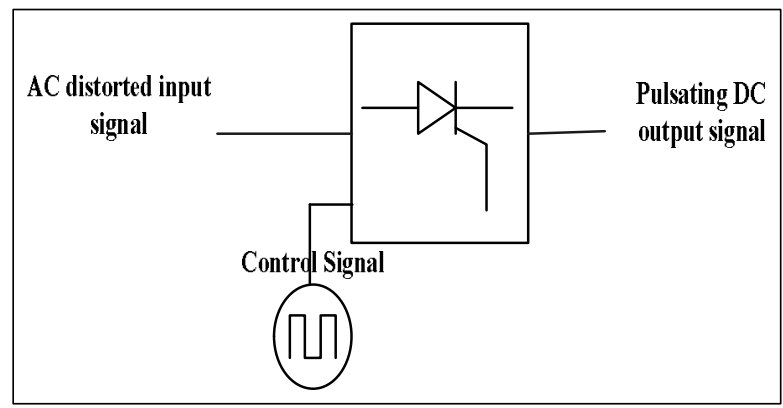

Fig. 1. AC/DC Thyristor Converter Block Diagram

Blake [7] gives a detailed account of the train pulse or rectangular function equation which is given in equation (5).

$$
S_{i}\left(\omega_{s} t\right)=\frac{\tau}{T}+\sum_{n=1}^{\infty} \frac{2}{\pi} \sin \frac{(n \pi \tau)}{T} \cos \left(n \omega_{s} t\right)
$$


Where $\tau$ : is the pulse width, $T$ is the sampling space or the period, and $\omega_{s}=2 \pi f_{s}$ is the sampling angular frequency. The frequency spectrum equation of the converter output DC pulsating current is described in equation (6).

$$
i_{o}(t)=I_{o} \cos \left(2 \pi f_{m} t\right)
$$

$I_{o}:$ is the average DC current, $2 \pi f_{m}$ is the angular frequency of the converter output signal. For accurate reconstruction of the input signal the Nyquist-Shannon condition should be met. This implies that the output current signal must be sampled at $f_{s}>2 f_{h}$. The following assumptions are thus made:

$$
\begin{aligned}
& 2 f_{h}=f_{i} \\
& f_{i}=f_{m} \\
& f_{s}=f_{m}+f_{i}
\end{aligned}
$$

This could also be rewritten in terms of the angular frequencies as follows:

$$
\omega_{s}=\omega_{m}+\omega_{i}
$$

Inserting (7) into (5) yields:

$$
S_{i}\left(\omega_{m} t+\omega_{i} t\right)=\frac{\tau}{T}+\sum_{n=1}^{\infty} \frac{2}{n \pi} \sin \frac{(n \pi \tau)}{T} \cos \left[n\left(\omega_{m}+\omega_{i}\right) t\right](8)
$$

Having met the Nyquist-Shannon condition, the distorted input signal can be accurately reconstructed. However for the purpose of harmonic frequency identification, the frequency spectrum equation of the distorted input signal should actually be more important rather than the continuous-time input signal. Equation (4) can therefore be applied as the product of equations (6) and (8) to describe the magnitude of current harmonic frequencies contained in the converter input phases.

$$
i_{i}(t)=i_{o}(t) \cdot S_{i}\left(\omega_{m} t+\omega_{i} t\right)
$$

Equation (9) yields the following:

$$
\begin{aligned}
& i_{i}(t)=\frac{\tau}{T} \sqrt{3} I_{o} \cos \omega_{m} t+ \\
& \sum_{m=0}^{\infty} \frac{2 \sqrt{3}}{\pi} I_{o} \cdot \frac{\sin (m p \pm 1) \frac{\pi \tau}{T}}{(m p \pm 1)} \cos \left[(m p \pm 1) \omega_{i} t+m p \omega_{m} t\right]
\end{aligned}
$$

Equation (10) can further be developed and expressed in terms of the thyristor firing and the conduction angles. This requires the following assumptions to be made:
1) Instantaneous commutation or no overlap condition between ingoing and outgoing thyristors;

2) The firing pulse should last for the entire actual conduction time.

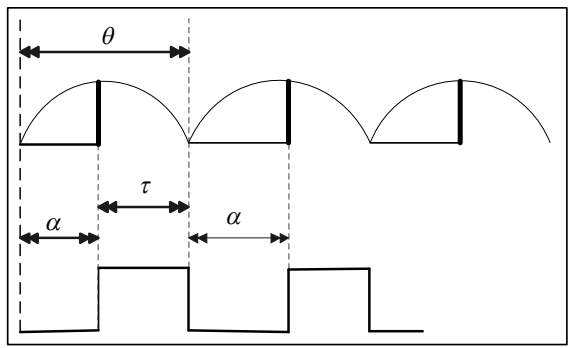

Fig. 2. Thyristor Pulse width, Firing and Conduction Angles

According to Fig.2 the following relationship does indeed apply between the pulse width, the firing and the conduction angle:

$$
\theta=\alpha+\tau
$$

Taking equation (11) into (10) produces:

$$
\begin{aligned}
& i_{i}(t)=\frac{(\theta-\alpha)}{T} \sqrt{3} I_{o} \cos \omega_{m} t+ \\
& \sum_{m=0}^{\infty} \frac{2 \sqrt{3}}{\pi} I_{o} \cdot \frac{\sin (m p \pm 1) \frac{\pi(\theta-\alpha)}{T}}{(m p \pm 1)} \cos \left[(m p \pm 1) \omega_{i} t+m p \omega_{m} t\right]
\end{aligned}
$$

It is now quite obvious that the current harmonic components contained in the reconstructed distorted input line equation can be evaluated on the basis of the firing angle.

\section{Evaluation of Harmonic Current Amplitudes}

\section{A. Numerical Evaluation}

The variations of the magnitude of the $(m p \pm 1)$ characteristic current harmonics, generated in the input-phase of the 6-pulse thyristor converter could be numerically assessed in terms of incremental change of the firing angle with the aid of the sampling theorem equations thus obtained. The impact of these variations on the individual harmonic distortion (IHD) for the following harmonic components: $5^{\text {th }}, 7^{\text {th }}, 11^{\text {th }}$ and $13^{\text {th }}$ at the following firing points; $10^{\circ}, 15^{\circ}, 20^{\circ}, 25^{\circ}$ and $30^{\circ}$ is also examined. To compute the HF of current harmonics, it is essential that the root mean square (RMS) of the harmonic current amplitudes $\left(A_{h}\right)$ obtained previously be determined and the actual value of the thyristor conduction angle which is the third of the full cycle [9]. From Fig. 2 the conduction cycle is repeated every half cycle, therefore the period (T) is 180 degrees or $\pi$. 
The RMS amplitude of the current harmonics could be expressed as:

$$
A_{h}=\frac{\sqrt{6}}{\pi} I_{o} \cdot \frac{\sin (m p \pm 1)\left(\frac{2 \pi}{3}-\alpha\right)}{(m p \pm 1)}
$$

The average DC output current $\left(I_{o}\right)$ is obtained by analysing the current path formed by the converter output and the DC motor resistance-inductance and the back electromotive force (RL-E) circuit which comprises the following parameters: the average DC output voltage $\left(\mathrm{V}_{\mathrm{DC}}\right)$, the back emf $(\mathrm{E})$, the armature resistance $(\mathrm{R})$ and inductance $(\mathrm{L})$. Assuming $10 \%$ of $\mathrm{V}_{\mathrm{DC}}$ is dropped across the armature resistance, the following equations are therefore applied:

$V_{D C}=\frac{3 \sqrt{2}}{\pi} V_{s} \cos \alpha \quad ; \quad E=(1-0.1) V_{D C}$

And

$$
I_{o}=\frac{V_{D C}-E}{\sqrt{R^{2}+(2 \pi f L)^{2}}}
$$

Where $\mathrm{V}_{\mathrm{s}}=380 \mathrm{~V} ; \mathrm{R}=2.5 \Omega ; \mathrm{L}=200 \mathrm{mH}$.

The percentage HF is therefore defined in the equation below:

$$
\% H F=\frac{A_{h}}{A_{1}} \times 100
$$

Where $A_{1}$ is the RMS amplitude of the fundamental current.

\section{B. MatLab/Simulink Simulation}

The DC VSD MatLab model (Fig. 3) is used to observe the behaviour of the amplitude of the characteristic harmonic currents induced in the input line per changing firing angle. The MatLab/Simulink 7.0 package provides the means of adjusting the thyristor firing angle through the synchronised 6-pulse generator which forms the control circuit of the converter system. It allows the circuit to be solved in continuous, sampled and hybridtime using the graphical user interface (GUI). The simulated circuit consists of: a three-phase source supplying a 6-pulse thyristor converter whose output terminals feed a DC motor. The connected DC motor is represented as an RL-E model. The fast Fourier transform (FFT) tool performed on the input line current of the converter by means of the software GUI at respective firing point is conducted to obtain the variation trends of harmonic amplitudes as well as the percentage individual harmonic distortion.

In addition to the parameters assigned to the DC motor RL-E model, the MatLab/Simulink equivalent circuit of the DC VSD further requires the supply system impedance (resistance and inductance) as well as the thyristor forward resistance to be assigned some operating values. These values are given as: $0.214 \Omega, 17$ $\mathrm{mH}$ and $0.001 \Omega$ respectively. In every simulation run, the FFT tool is invoked to determine the magnitude of targeted current harmonics present in the converter input line.

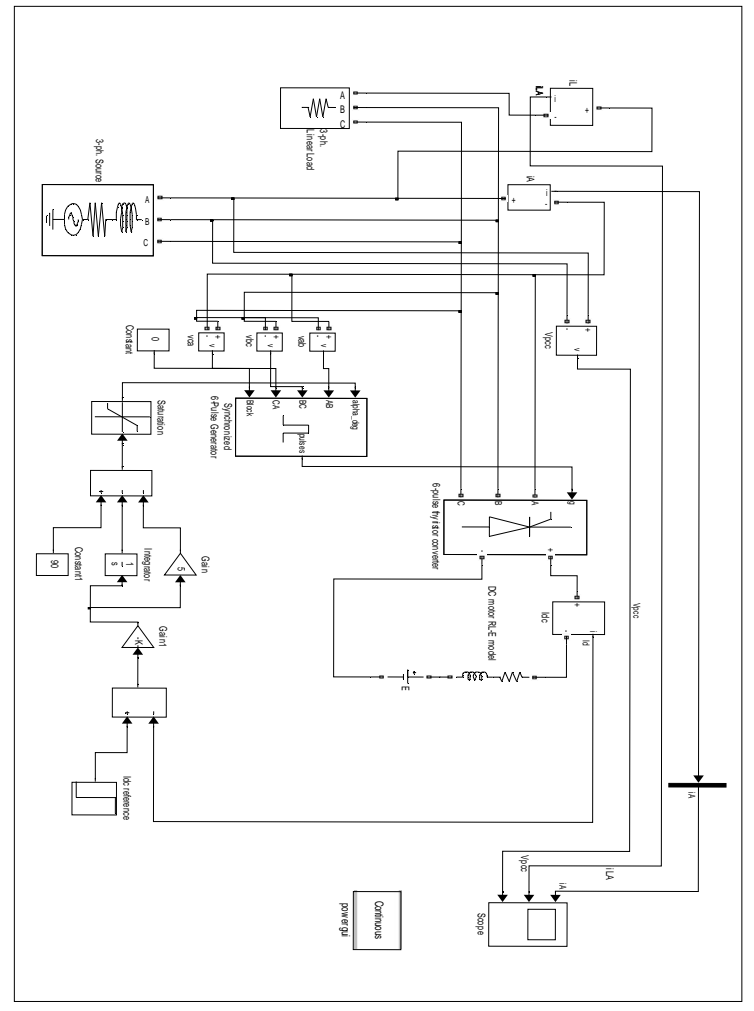

Fig. 3. MatLab/Simulink Equivalent Circuit of the DC VSD

\section{Results}

The variations of the percentage harmonic factor for the characteristic harmonic currents against the firing angle obtained with the sampling theorem method as well as with the MatLab/Simulink simulation are presented Fig. 4 and Fig. 5 respectively.

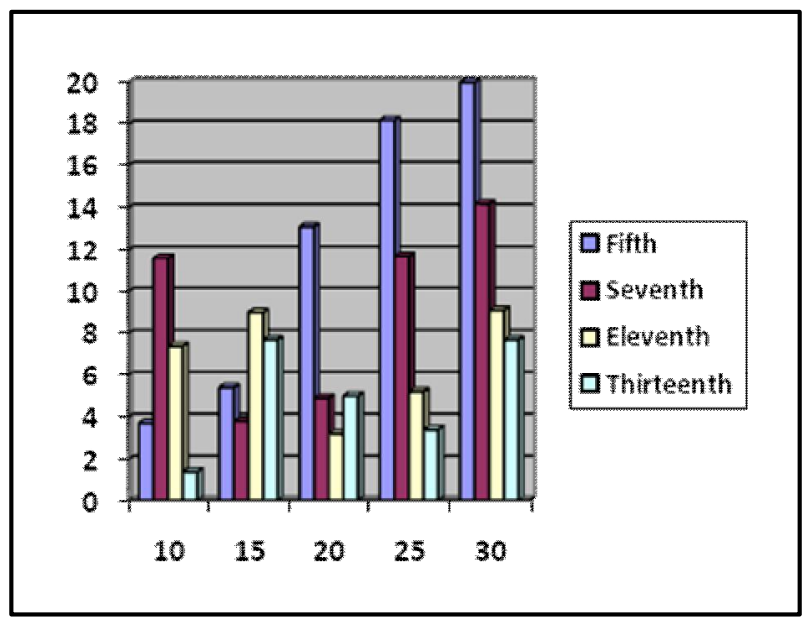

Fig. 4. Percentage HF versus Firing Angle (Sampling Theorem) 


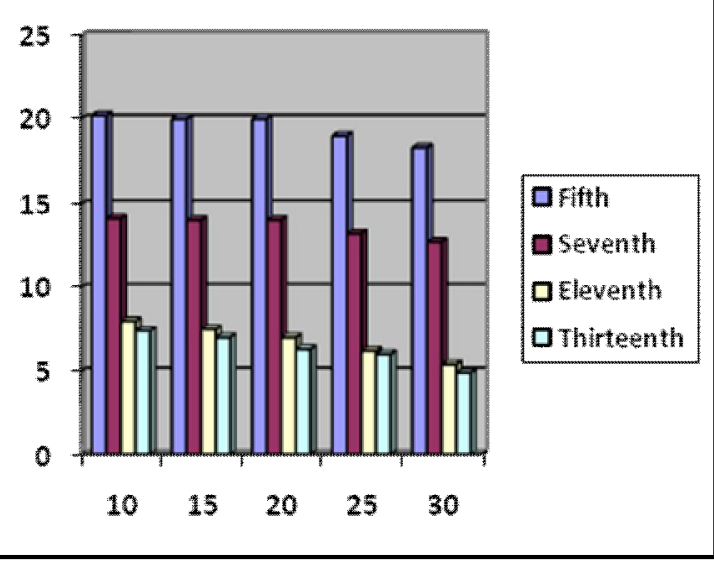

Fig. 5. Percentage HF versus Firing Angle (MatLab/Simulink)

The trends generated when the sampling theorem (Fig. 4) has been applied show that the percentage harmonic factor of the current harmonics does not match the simulated trend. The percentage HF obtained generally increases with an increase of the firing angle. However, at some points an alternating of growth and decay pattern is observed before any consistent growth while the firing angle is increasingly adjusted. This could be attributed to the dependency of the sampling theorem equations on the sine function. Further studies are therefore required to bridge this gap.

\section{Conclusion}

This study tests the validity of the sampling theorem in the analysis of the input current harmonics in 6-pulse thyristor converters on the basis of the change in percentage harmonic factor per firing angle adjustment. The results obtained generally seem to be in contradiction with the trend obtained using the MatLab/Simulink. In the light of this, the sampling theorem as applied to this work requires further refinements to be deemed adequate for converter line current harmonic analysis in 6-pulse thyristor converters.

\section{References}

[1] K.Zdrozis, "Mathematical Model of 4-Quadrant DC Electric Drive with 6-pulse Dual Converter", American Journal of Applied Sciences, Science Publications 2005, pp. 1025- 1027.

[2] M. Rashid, Power Electronics-Circuits, Devices and Applications, Prentice Hall, 2004, pp. 304- 640.

[3] J. Arillaga and N. Watson, "The Harmonic Domain Revisited", $13^{\text {th }}$ ICHQP2008, pp. 166-174.

[4] T. Mumcu, K. Gulez and M. Mercimek, "Switching Control of an AC/DC Converter by Neural Netwoks", International Journal of Information Technology, 2009, pp. 78-86.

[5] I. Selesnick, "Interpolating Multiwavelet Bases and the Sampling Theorem", IEEE Transactions on signal Processing, 1999, Vol. 47, No.6, pp. 1615-1621.

[6] P. Vaidyanathan, "Generalisations of the Sampling
Theorem: Seven Decades after Nyquist", IEEE Transactions On Circuits and Systems, 2001, Vol. 48, No.9, pp. 1094 1109.

[7] R. Blake, Electronic Communication Systems, Delmar Thomson Learning, 2001, pp.286-309.

[8] G.Chang and Task Force, "Characteristics and Modelling of Harmonic Sources-Power Electronic Devices", IEEE Transactions on Power Engineering, 2001, Vol. 21, No.8, pp. 62-63.

[9] A. Ahmed, Power Electronics for Technology, Pearson Education, 2003, pp. 74-265. 